\title{
TOXICIDADE DE SOLO DE “LANDFARMING” DE REFINARIA DE PETRÓLEO TRATADO COM ALGAS PARA A MINHOCA Eisenia fetida
}

\section{'LANDFARMING' SOIL TOXICITY FROM OIL REFINERY TREATED WITH ALGAE FOR THE EARTHWORM Eisenia fetida}

\section{Acácio Aparecido Navarrete ${ }^{1}$; Marcos Aparecido Pizano²; Orlando Necchi Júnior ${ }^{3}$, Antônio Carlos Simões Pião ${ }^{4}$; Dejanira de Franceschi de Angelis ${ }^{1}$}

\begin{abstract}
${ }^{1}$ Departamento de Bioquímica e Microbiologia, Instituto de Biociências, UNESP, Rio Claro, Brasil - navarret@esalq.usp.br, dangelis@rc.unesp.br

${ }^{2}$ Departamento de Ecologia, Instituto de Biociências, UNESP, Rio Claro, Brasil mapizano@rc.unesp.br

${ }^{3}$ Departamento de Zoologia e Botânica, Instituto de Biociências, Letras e Ciências

Exatas, UNESP, São José do Rio Preto, Brasil - orlando@ibilce.unesp.br

${ }^{4}$ Departamento de Estatística, Matemática Aplicada e Computação, Instituto de

Geociências e Ciências Exatas, UNESP, Rio Claro, Brasil - piao@rc.unesp.br
\end{abstract}

\section{RESUMO}

O "landfarming" é uma técnica de biorremediação muito utilizada para o tratamento de resíduos da indústria petroquímica. Neste estudo, utilizou-se Eisenia fetida para verificar a destoxicação do solo de "landfarming" de resíduos petroquímicos por comunidades de algas alóctones. Amostras do solo foram coletadas a $0-20 \mathrm{~cm}$ no "landfarming" da Refinaria de Paulínia (REPLAN/PETROBRAS) e inoculadas, sob condições controladas, com algas provenientes de solo. Decorridos 30 dias da inoculação, membros das comunidades com crescimento ativo foram identificados ao nível gênero: Anabaena, Chroococcus, Scytonema e Lyngbya (Cyanophyceae), Klebsormidium, Mougeotia e Oedogonium (Chlorophyceae) e Navicula (Bacillariophyceae). A toxicidade do solo de "landfarming" exposto ao crescimento de algas foi avaliada para a minhoca $E$. fetida antes da inoculação e após 180 dias do estabelecimento das comunidades de algas. Foi considerado na avaliação o efeito da incorporação e remoção do crescimento algal visível na toxicidade deste solo. A análise da toxicidade não revelou efeito agudo para a minhoca $E$. fetida. No entanto, o efeito na biomassa de espécimes 
adultos da minhoca constatou destoxicação do solo de "landfarming" quando submetido à remoção do crescimento de algas visível, após 180 dias da inoculação. Os resultados deste estudo indicam que efeitos subletais na biomassa da minhoca $E$. fetida são capazes de fornecer medidas efetivas para o monitoramento da toxicidade durante o processo de remediação do solo de "landfarming" de refinaria de petróleo. Palavras-chave: Eisenia fetida. Ecotoxicidade. "Landfarming". Refinaria de petróleo

\section{ABSTRACT}

Landfarming is a bioremediation technique to remediate soils contamined for residual treatment from petrochemical industry. In this study, Eisenia fetida was used to verify the landfarming soil detoxication from petrochemical sludges by allochthonous algal community. Soil samples were collected at $0-20 \mathrm{~cm}$ in landfarming Paulínia oil refinery (REPLAN/PETROBRAS) and inoculated, under controlled conditions, with algae from non-contaminated soil. After 30 days of inoculation, community members with active growth were identified at the genus level: Anabaena, Chroococcus, Scytonema and Lyngbya (Cyanophyceae), Klebsormidium, Mougeotia and Oedogonium (Chlorophyceae) and Navicula (Bacillariophyceae). The landfarming soil toxicicity exposed to the algal growth were evaluated for the earthworm E. fetida before the inoculation and after 180 days algal community establishment. The incorporation and the removal effect of visible algal growth in the toxicity of this soil were evaluated. The toxicity analysis did not revealed acute effect for the earthworm E. fetida. However, the biomass effect of adult earthworms specimens showed detoxication of the landfarming soil when submitted to removal of the visible algal growth, after 180 days of inoculation. The results of this study indicated that sublethals effects of the E. fetida earthworm biomass are able to predict effective measurements for monitoring soil toxicity during the process of remediation of landfarming soil from oil refinery.

Keywords: Eisenia fetida. Ecotoxicicity. Landfarming. Oil refinery

\section{INTRODUÇÃO}

O petróleo representa mundialmente a principal fonte de combustível e sua tecnologia de refino, semelhante a outros processos industriais de larga escala, se apresenta como fonte potencial de poluição (URURAHY et al., 1998). Na busca de minimizar os problemas apresentados quanto à deposição ou reutilização dos resíduos gerados na etapa de refino do petróleo, empregam-se tecnologias inovadoras de tratamento, as quais contemplam principalmente a utilização de remediação através de biopilhas, bioventilação e "landfarming" (USEPA, 2001). 
Dentre os numerosos sistemas de biotratamento, o "landfarming" utiliza a atividade dos microrganismos do solo para degradar, modificar ou imobilizar os vários componentes dos resíduos oleosos. Na indústria do petróleo, esta tecnologia de remediação diminui as concentrações dos constituintes do petróleo mediante a biodegradação nas camadas superficiais do solo envolvendo espalhamento, escarificação em camadas finas sobre a superfície do terreno e estimulação da atividade microbiana aeróbia pela aeração e adição de minerais, nutrientes e umidade (USEPA, 2001). Apesar de pouco difundido no Brasil, este sistema tem sido usado pela indústria do petróleo nacional, devido a sua simplicidade, baixo custo operacional e eficácia perante as condições naturais tropicais.

O monitoramento do "landfarming" nas áreas industriais tem sido realizado apenas com o auxílio de atributos químicos do solo, sem a inserção de avaliações ecotoxicológicas. Por meio dos ensaios ecotoxicológicos, entretanto, são verificados os efeitos das variáveis ambientais que são capazes de afetar a toxicidade das substâncias aos componentes vivos de um ecossistema. Para isto, têm sido desenvolvidas metodologias padronizadas a fim de determinar os efeitos agudos e crônicos em organismos-teste com base na mortalidade, genotoxicidade, mutagenicidade e respostas subletais como reprodução, comportamento e crescimento (AVILA et al., 2007; SOUZA et al., 2009).

Os ensaios para avaliação da ecotoxicidade com organismos de solo não são bem estabelecidos e descritos no Brasil por não existirem ainda normas atualizadas na legislação brasileira. Dessa forma, quando há necessidade de avaliação da contaminação de amostras de solos, são frequentemente utilizados métodos internacionalmente reconhecidos, como os da ISO (International Organization for Standardization) (ISO, 2002), OECD (Organization for Economic Co-operation and Development) (OECD, 1984) e USEPA (United States Environmental Protection Agency) (USEPA, 1994).

Estes métodos, entretanto, são desenvolvidos para determinar a toxicidade de substâncias adicionadas a um substrato artificial, a fim de que vários interferentes sejam eliminados. O grande desafio na adaptação desses métodos para a complementação da avaliação de áreas contaminadas é a substituição do substrato artificial por amostras de solos oriundas do ambiente natural (SISINNO et al., 2006).

Spurgeon e Hopkin $(1995,1996)$ asseguram que os organismos-teste são menos sensíveis a solos de áreas contaminadas do que os substratos contaminados artificialmente e atribuem esta condição à maior biodisponibilidade de elementos nestes últimos. Avila et al. (2007), ao empregarem amostras de solos provenientes de áreas contaminadas em estudos de toxicidade, afirmam, com base em seus resultados, não estar clara a forma como a biodisponibilidade dos elementos é mais adequada para determinar efeitos sobre as respostas dos bioensaios de toxicidade.

A minhoca Eisenia fetida tem se mostrado muito eficaz na avaliação da qualidade do solo, sendo facilmente mantida em condições laboratoriais. Esse organismo apresenta alta taxa reprodutiva, com tempo de maturação de sete a oito 
semanas, facilidade de coleta em fontes naturais e estreita relação com diferentes compartimentos do solo. Pode ser também uma fonte de recurso para diversos organismos, além de ingerir grande quantidade de solo, tendo importância na cadeia trófica. Em bioensaios foi possível obter dados de crescimento, sobrevivência e reprodução desse organismo (ASTM, 1995).

Assim, o objetivo deste trabalho foi avaliar o potencial de destoxicação do solo de "landfarming" da Refinaria de Paulínia (REPLAN/PETROBRAS) inoculado com comunidades de algas alóctones mediante análise de alterações na biomassa de espécimes adultos de E. fetida.

\section{MATERIAL E MÉTODOS}

A amostragem do solo foi realizada de acordo com a CETESB (1984) no "landfarming" da Refinaria de Paulínia, Paulínia, SP. O solo de "landfarming" foi peneirado em malha de $1,19 \mathrm{~mm}$ de abertura, sendo transferido o volume de $1,5 \mathrm{~L}$ para 45 bandejas plásticas de $11,5 \mathrm{~cm}$ x $8,25 \mathrm{~cm} \times 3,0 \mathrm{~cm}$, constituindo o substrato para o crescimento das comunidades de algas.

Cultivo das comunidades de algas - O inóculo algal foi elaborado a partir de 10 sub-amostras coletadas aleatoriamente em microhabitats estabelecidos na superfície do solo interna e externamente à Casa de Vegetação no Jardim Experimental do Instituto de Biociências da UNESP, Campus de Rio Claro, SP. Após maceração do material coletado, acrescentou-se água à mistura até o volume de $4,5 \mathrm{~L}$ e borrifaram-se alíquotas de $150 \mathrm{~mL}$ sobre 30 unidades experimentais contendo solo de "landfarming". Outras 15 unidades experimentais foram privadas da inoculação, constituindo-se assim o conjunto controle. A manutenção da saturação hídrica dos substratos de crescimento das comunidades de algas foi efetuada por sistema de irrigação por microaspersão, em dias com elevada taxa de evaporação e com o uso de um borrifador de pressão manual nos dias em que se constatou pouca variação do nível de saturação hídrica do solo.

Coleta e identificação das comunidades de algas - Após 30 dias da inoculação algal nos substratos de crescimento, camadas gelatinosas, algas filamentosas e material crustoso foram coletados com o auxílio de espátula e pinça na profundidade de 0,5 a $3,0 \mathrm{~cm}$, sendo transferidos para frascos de vidro de $10 \mathrm{~mL}$ contendo formol a $4 \%$. Apenas os gêneros de algas que revelaram crescimento ativo no momento inicial da colonização foram identificados mediante observação direta e análise em microscópio óptico da superfície dos solos emulsificada com água.

Estimativa visual do crescimento das comunidades de algas - O percentual de cobertura da superfície das unidades experimentais pela biomassa algal foi estimado em intervalos de 30 dias a partir da inoculação. Para tanto, empregou-se um filme plástico transparente de área correspondente, em forma e extensão, à superfície das unidades experimentais. A área total do filme plástico foi dividida em quatro 
quadrantes de área equivalente a $25 \%$ da área total, de modo a possibilitar uma estimativa visual do recobrimento da superfície do solo pelas comunidades de algas.

Preparo dos substratos para utilização em avaliação toxicológica - Após 180 dias da inoculação de comunidades de algas alóctones, foram selecionadas ao acaso oito unidades experimentais que receberam inóculo algal e quatro unidades experimentais privadas da inoculação. Das oito unidades experimentais selecionadas contendo o inóculo, quatro tiveram o crescimento algal visível incorporado ao substrato e quatro tiveram o crescimento algal visível removido da superfície do substrato. Efetuado o processamento descrito, as unidades experimentais foram privadas do suprimento hídrico sendo mantidas internamente à Casa de Vegetação. Após complementação da secagem do substrato em estufa a $50^{\circ} \mathrm{C}$, procedeu-se a maceração e a disposição do mesmo para a avaliação toxicológica.

Avaliação toxicológica com Eisenia fetida - A criação do organismo-teste procedeu-se de acordo com o Manual de Testes para Avaliação da Ecotoxicidade de Agentes Químicos (BRASIL, 1988). O lote dos organismos utilizados foi avaliado por meio de ensaio com sílica. A concentração letal média $\left(\mathrm{CL}_{50}\right)$ da sílica manteve-se entre $35 \mathrm{mg} \mathrm{kg}^{-1}$ e $160 \mathrm{mg} \mathrm{kg}^{-1}$ de peso seco da minhoca. Foram usados indivíduos adultos de E. fetida, com clitelo desenvolvido e peso entre 300 e $600 \mathrm{mg}$. Populações de 10 indivíduos foram formadas, lavadas sob jato de água corrente e enxugadas cuidadosamente em papel absorvente. $O$ peso inicial de cada população foi conferido em balança (AS $2000 \mathrm{C}+/-0,01 \mathrm{~g}$ ) e os indivíduos foram transferidos para sacos plásticos $(1,5 \mathrm{~L})$ previamente perfurados com o auxílio de alfinete, contendo o substrato a ser testado $(350 \mathrm{~g})$ homogeneizado com $90 \mathrm{~mL}$ de água destilada e $500 \mathrm{~g}$ de bolas de vidro de $1,5-2,0 \mathrm{~cm}$ de diâmetro. $O$ meio de criação das populações de E. fetida foi utilizado como solo controle no ensaio realizado. Todos os sacos plásticos foram lacrados e mantidos por 14 dias em incubadora de DBO sob temperatura estável de $20 \pm 2^{\circ} \mathrm{C}$, privada de iluminação. Decorrido o tempo de teste, os sacos plásticos foram abertos e as populações lavadas sob jato de água corrente, enxugadas cuidadosamente em papel absorvente e pesadas em balança. As diferenças entre o peso inicial e o peso final das populações de E. fetida foram submetidas à análise e interpretação fatorial com três fatores.

Avaliação química - Os atributos químicos do solo de "landfarming" submetido aos diferentes tratamentos foram analisados pelo Instituto Campineiro de Análise de Solo e Adubo, Campinas, SP e pelo Laboratório Agrotécnico de Piracicaba, Piracicaba, SP. Os extratos solubilizados foram analisados pelo Laboratório de Hidrogeologia e Hidrogeoquímica do Instituto de Geociências e Ciências Exatas da UNESP, Rio Claro, SP. 


\section{RESULTADOS E DISCUSSÃO}

Os gêneros de algas com maior abundância relativa identificados no início da colonização foram: Anabaena, Chroococcus, Scytonema e Lyngbya (Cyanophyceae), Klebsormidium, Mougeotia e Oedogonium (Chlorophyceae) e Navicula (Bacillariophyceae). Além destes, observou-se a presença de esporos algais.

A estimativa visual do crescimento das comunidades de algas revelou $100 \%$ de cobertura da superfície pela biomassa algal, transcorridos 30 dias da inoculação nas unidades experimentais com solo de "landfarming", mantendo-se o recobrimento durante os 180 dias de avaliação.

Análises químicas mostraram diminuição da concentração de $\mathrm{Cd}, \mathrm{Cu}, \mathrm{Pb}, \mathrm{Zn}$ e $\mathrm{N}$ e aumento de matéria orgânica (MO) e P no solo de "landfarming" submetido a remoção do crescimento algal visível após 180 dias da inoculação (Tabela 1). Nos extratos solubilizados do mesmo solo constatou-se diminuição dos valores de $\mathrm{Zn}$ e $\mathrm{Pb}$, enquadrando-os abaixo do limite máximo especificado pela ABNT NBR 10004:2004 (ABNT, 2004).

TABELA 1 - Características químicas de amostras do solo de "landfarming" e de extratos solubilizados analisadas após 0 e 180 dias da inoculação algal.

\begin{tabular}{|c|c|c|c|c|c|c|c|c|c|c|c|c|}
\hline \multirow{3}{*}{$\begin{array}{l}\text { Dias após a } \\
\text { inoculação }\end{array}$} & \multirow{3}{*}{ Inoculação } & \multirow{3}{*}{ Processamento } & \multicolumn{8}{|c|}{ Solo } & \multirow{2}{*}{\multicolumn{2}{|c|}{$\frac{\text { extrato solubilizado }}{\mathrm{mg} \mathrm{L}^{-1}}$}} \\
\hline & & & \multirow{2}{*}{$\begin{array}{c}\mathrm{CaCl}_{2} \\
\mathrm{pH}^{\mathrm{a}}\end{array}$} & \multirow{2}{*}{$\begin{array}{c}\mathrm{g} \mathrm{dm}^{-3} \\
\mathrm{MO}^{\mathrm{a}}\end{array}$} & \multirow{2}{*}{$\begin{array}{c}\mathrm{g} \mathrm{Kg}^{-1} \\
\mathrm{~N}^{\mathrm{b}}\end{array}$} & \multirow{2}{*}{$\begin{array}{c}\mathrm{mg} \mathrm{dm}^{-3} \\
\mathrm{P}^{\mathrm{a}}\end{array}$} & \multicolumn{4}{|c|}{ Ppm } & & \\
\hline & & & & & & & $\mathrm{Cd}^{\mathrm{a}}$ & $\mathrm{Cu}^{\mathrm{a}}$ & $\mathrm{Pb}^{\mathrm{a}}$ & $\mathrm{Zn}^{\mathrm{a}}$ & $\mathrm{Pb}^{\mathrm{c}}$ & $\mathrm{Zn}^{\mathrm{c}}$ \\
\hline \multirow[t]{3}{*}{0} & & & 5,5 & 106 & 4,55 & 42,0 & 2,48 & 3,0 & 109,80 & 75,0 & 0,013 & 6,70 \\
\hline & com & incorporação & 5,9 & 185 & 5,39 & 62,0 & 2,49 & 8,6 & 110,77 & 155,0 & $<0,010$ & 0,042 \\
\hline & inóculo & remoção & 5,9 & 132 & 0,35 & 50,0 & 2,18 & 2,4 & 102,32 & 64,0 & $<0,010$ & 0,011 \\
\hline 180 & $\begin{array}{c}\text { sem } \\
\text { inóculo }\end{array}$ & & 5,9 & 105 & 4,55 & 45,0 & 2,42 & 3,1 & 110,20 & 98,0 & 0,011 & 0,37 \\
\hline
\end{tabular}

Limite máximo no extrato solubilizado ${ }^{d}$

abtido pelo ICASA - Instituto Campineiro de Análise de Solo e Adubo

bobtido pelo Pirasolo - Laboratório Agrotécnico de Piracicaba

cobtido pelo Laboratório de Hidrogeologia e Hidrogeoquímica do Instituto de Geociências e Ciências Exatas (IGCE/UNESP)

${ }^{\mathrm{d}}$ ABNT NBR 10004:2004

$\mathrm{Na}$ avaliação da toxicidade não foi constatada mortalidade de E. fetida no solo de "landfarming". Não sendo observado o efeito agudo nos diferentes tratamentos, o efeito na biomassa da minhoca $E$. fetida foi avaliado (Tabela 2). Os valores das diferenças entre o peso inicial e o peso final das populações de E. fetida foram convertidos em percentual de perda de peso e submetidos à análise e interpretação fatorial com 3 fatores: SOLO (solo de "landfarming"/meio de criação), INOCULAÇÃO (com inóculo/sem inóculo) e PROCESSAMENTO (remoção/incorporação do crescimento algal visível), indicando efeito significativo dos fatores $(p<0,05)$ na biomassa da minhoca. 
TABELA 2 - Peso inicial e peso final de populações de E. fetida submetidas aos fatores de variação SOLO, INOCULAÇÃO e PROCESSAMENTO.

\begin{tabular}{|c|c|c|c|c|c|c|}
\hline & $\begin{array}{l}\text { Dias após a } \\
\text { inoculação }\end{array}$ & Inoculação & Processamento & Peso inicial & Peso final & Diferença \\
\hline $\begin{array}{l}\text { Solo } \\
\text { controle }\end{array}$ & & & & 4,877 & 4,122 & 0,755 \\
\hline $\begin{array}{c}\text { Solo } \\
\text { landfarming }\end{array}$ & $\begin{array}{c}0 \\
180\end{array}$ & $\begin{array}{l}\text { com inóculo } \\
\text { sem inóculo }\end{array}$ & $\begin{array}{l}\text { remoção } \\
\text { incorporação }\end{array}$ & $\begin{array}{l}4,698 \\
4,870 \\
4,620 \\
4,712 \\
\end{array}$ & $\begin{array}{l}0,749 \\
3,047 \\
1,976 \\
1,007 \\
\end{array}$ & $\begin{array}{l}3,949 \\
1,823 \\
2,644 \\
3,705 \\
\end{array}$ \\
\hline
\end{tabular}

valores médios de 4 réplicas

A análise de variância dos valores referentes ao percentual de perda de peso das populações de $E$. fetida testada para o modelo fatorial, considerando como fatores de variação SOLO, INOCULAÇÃO e PROCESSAMENTO, revelou ao nível de 5\% de significância, diferença na biomassa da minhoca E. fetida para as interações SOLO X INOCULAÇÃO $(p=0,0001)$ e SOLO X INOCULAÇÃO $X$ PROCESSAMENTO $(\mathrm{p}=0,0057)$.

Aplicando o teste de Tukey nos resultados obtidos, verificou-se que as diferenças no percentual de perda de peso foram estatisticamente significativas e que os menores valores ocorreram para o solo de "landfarming" que recebeu inóculo algal e teve o crescimento algal visível removido da superfície do substrato após 180 dias da inoculação (Tabela 3).

TABELA 3 - Resultados da aplicação do teste de Tukey para os fatores de variação SOLO, INOCULAÇÃO e PROCESSAMENTO.

\begin{tabular}{lcccccc}
\hline & \multicolumn{2}{c}{ Solo } & \multicolumn{2}{c}{ Inoculação } & \multicolumn{2}{c}{ Processamento } \\
& landfarming & meio de criação & sem inóculo & com inóculo & incorporação & remoção \\
\hline Grupo Tukey & $A^{*}$ & $B^{*}$ & $A^{*}$ & $B^{*}$ & $A^{*}$ & $B^{*}$ \\
Média & 2,51313 & 0,43250 & 2,29688 & 1,84875 & 2,37063 & 1,77500 \\
& & & & & &
\end{tabular}

* Diferença estatística

Os gêneros de algas com crescimento ativo no início da colonização ocorreram com abundância relativa diferenciada nas comunidades estabelecidas na superfície das unidades experimentais. Segundo Lund (1946), as espécies dominantes aparecem nos primeiros dias e permanecem dominantes na superfície do solo durante alguns meses, embora existam pequenas flutuações nas comunidades.

A diminuição da concentração de metais pesados nas amostras do solo de "landfarming" tratado com algas e submetidas à remoção do crescimento algal visível após 180 dias da inoculação pode estar relacionada com o desempenho de diferentes espécies de algas na captação e acumulação de íons metálicos, como 
evidenciado por Hao et al. (1994), Rollemberg et al. (1998) e Godlewska-Zylkiewicz (2001), sendo dependente do desenvolvimento da biomassa algal e do pH da solução.

Em todos os tratamentos analisados constatou-se perda de peso das populações de E. fetida, inclusive no solo controle. As diferenças entre o peso inicial e o peso final, no entanto, não foram às mesmas, para os fatores de variação SOLO, INOCULAÇÃO e PROCESSAMENTO.

O desvio padrão (SQ) calculado para o fator de variação SOLO (SQ = 6,20400), sem interação com outros fatores de variação, revelou diferença no percentual de perda de peso de E. fetida para o solo de "landfarming" e o meio de criação de populações da minhoca, evidenciando interação negativa entre os elementos do solo de "landfarming" e os organismos associados às suas condições naturais. Esta diferença, detalhada pelo teste de Tukey, diminuiu seu valor mediante inoculação de comunidades de algas alóctones no solo de "landfarming" e remoção do crescimento algal visível após 180 dias da adição do inóculo.

A variação no peso corpóreo de espécimes da minhoca $E$. fetida é um efeito subletal verificado por muitos autores ao analisarem a contaminação de amostras de solos artificiais e naturais. Spurgeon et al. (1994) constataram declínio do peso de populações de E. fetida expostas a uma série de diferentes concentrações de $\mathrm{Cd}, \mathrm{Cu}$, $\mathrm{Pb}$ e $\mathrm{Zn}$ em solos artificiais. Shin e Kim (2001) também constataram variações no peso corpóreo de espécimes E. fetida ao empregá-la como organismo-teste no monitoramento do processo de remediação de solos contaminados com hidrocarbonetos.

Neuhauser e Callahan (1989) verificaram o efeito de concentrações subletais de compostos químicos no crescimento e reprodução de populações de E. fetida e constataram que a variação no peso da população pode atuar como um indicador de efeitos destrutivos em processos do solo. Deve-se ressaltar, entretanto, que variáveis relacionadas à composição do solo a ser testado precisam ser sempre bem avaliadas, se o objetivo dos ensaios envolver a avaliação da toxicidade com amostras naturais, sem a necessidade de mistura com o solo artificial.

Assim, a verificação de respostas subletais na biomassa de populações de $E$. fetida ao longo de 14 dias mostrou-se capaz de fornecer medidas efetivas para o monitoramento da toxicidade do solo durante o processo de tratamento com algas avaliado em amostras do "landfarming" da Refinaria de Paulínia (REPLAN/PETROBRAS).

\section{AGRADECIMENTOS}

Os autores agradecem ao Programa de Recursos Humanos (PRH-05) da Agência Nacional do Petróleo (ANP), pelo apoio financeiro e a Refinaria de Paulínia (REPLAN/PETROBRAS) pela concessão do solo de "landfarming". 


\section{REFERÊNCIAS}

ABNT - ASSOCIAÇÃO BRASILEIRA DE NORMAS TÉCNICAS. Resíduos sólidos - Classificação, ABNT NBR 10004:2004, 2004.

ASTM - AMERICAN SOCIETY FOR TESTING AND MATERIALS. Standard Guide for Conducting a Laboratory Soil Toxicity Test with Lumbricid Earthworm Eisenia foetida, ASTM E1676-95, 1995.

AVILA, G.; GAETE, H.; MORALES, M. Reproducción de Eisenia foetida en suelos agrícolas de áreas mineras contaminadas por cobre y arsénico. Pesquisa Agropecuária Brasileira, Brasília, v.42, n.3, 435-441, 2007.

BRASIL. Ministério do Interior. Secretaria Especial do Meio Ambiente. Avaliação da toxicidade para organismos do solo: minhoca Eisenia foetida. In: MANUAL de testes para avaliação da ecotoxicidade de agentes químicos. Brasília, D5: 1-7, 1988.

CETESB - COMPANHIA DE TECNOLOGIA E SANEAMENTO AMBIENTAL. Solos - Coleta e preparação de amostras - Procedimentos. Norma Técnica L6.245, São Paulo, 1984.

GODLEWSKA-ZYLKIEWICZ, B. Analytical applications of living organisms for preconcentration of trace metals and their speciation. Critical Reviews in Analytical Chemistry, Londres, v.31, n.3, 175-189, 2001.

HAO, Y.; ZHAO, Y.; RAMELOW, G.L. Uptake of metal ions by nonliving biomass derived from marine organisms-effect of $\mathrm{pH}$ and chemical treatments. Journal of Environmental Science and Health, Philadelphia, v. 29, 2235-2254, 1994.

ISO (INTERNATIONAL ORGANIZATION FOR STANDARDIZATION). Draft Avoidance test for testing the quality of soils and the toxicity of chemicals - Part 1: Test with earthworms (Eisenia fetida), 2002.

LUND, J.W.G. Observations on soil algae. The ecology, size, and taxonomy of British soil diatoms. New Phytologist, Lancaster, v.45, n.1, 196-219, 1946.

NEUHAUSER, E.F.; CALLAHAN, C.A. Growth and reproduction of the earthworm Eisenia fetida exposed to sublethal concentrations of organic chemicals.

Soil Biology \& Biochemistry, Oxford, v.22, 175-179, 1990. 
OECD (ORGANIZATION FOR ECONOMIC COOPERATION AND DEVELOPMENT). Guideline for testing of chemicals: Earthworm, acute toxicity test, No. 207, 1984.

ROLLEMBERG, M.C.; SIMÕES GONÇALVES, M.L.S.; CORREIA DOS SANTOS, M.M.; BOTELHO, M.J. Thermodynamics of uptake of cadmium by Chlorella marina. Bioelectrochemistry and Bioenergetics, Lausanne, v.48, 61-68, 1999.

SISINNO, C.L.S.; BULUS, M.R.M.; RIZZO, A.C.; MOREIRA, J.C. Ensaio de comportamento com minhocas (Eisenia fetida) para avaliação de áreas contaminadas: resultados preliminares para contaminação por hidrocarbonetos. Journal of the Brazilian Society of Exotoxicology, Rio Grande, v.1, n.2, 137-140, 2006.

SHIN, K.; KIM, K. Ecotoxicity monitoring of hydrocarbon-contaminated soil using earthworm (Eisenia foetida). Environmental Monitoring and Assessment, Bengal, v.70, 93-103, 2001.

SOUZA, T.S.; HENCKLEIN, F.A.; ANGELIS, D.F.; GONÇALVES, R.A.; FONTANETTI, C.S.; The Allium cepa bioassay to evaluate landfarming soil, before and after the addition of rice hulls to accelerate organic pollutants biodegradation. Ecotoxicology and Environmental Safety, Munique, v.72, n.5, 1363-1368, 2009.

SPURGEON, D.J.; HOPKIN, S.P.; JONES, D.T. Effects of Cadmium, Copper, Lead and Zinc on growth, reproduction and survival of the earthworm Eisenia fetida (Savigny): assessing the environmental impact of point-source metal contamination in terrestrial ecosystems. Environmental Pollution, The Netherlands, v.84, 123-130, 1994.

SPURGEON, D.J.; HOPKIN, S.P. Extrapolation of the laboratory-based OECD earthworm toxicity test to metal-contaminated field sites. Ecotoxicology, The Netherlands, v.4, n.3, 190-205, 1995.

SPURGEON, D.J.; HOPKIN, S.P. Effects of metal-contaminated soils on the growth, sexual development, and early cocoon production of the earthworm Eisenia foetida, with particular reference to zinc. Ecotoxicology and Environmental Safety, Munique, v.35, 86-95, 1996.

URURAHY, A.F.P.; PEREIRA JR., N.; MARTINS, M.D.M. Desempenho de um biorreator do tipo CSTR no processo de degradação de borra oleosa. Boletim Técnico Petrobrás, Rio de Janeiro. jul/dez, v.41, 125-132, 1998. 
USEPA (UNITED STATES ENVIRONMENTAL PROTECTION AGENCY). Treatment technologies screening matrix and reference guide: version 4.0. Washington, 2001.

USEPA (UNITED STATES ENVIRONMENTAL PROTECTION AGENCY). Catalogue of standard toxicity tests for ecological risk assessment. ECO update, intermittent bulletin, volume 2, number 2. EPA 540/F-94/013, 1994. 